OPEN ACCESS

Edited by:

Akio Adachi,

Kansai Medical University, Japan

Reviewed by:

Tong-Qing An,

Harbin Veterinary Research Institute (CAAS), China

Toyoyuki Ose,

Hokkaido University, Japan

*Correspondence:

Hongying Chen

chenhy@nwafu.edu.cn

Specialty section:

This article was submitted to

Virology,

a section of the journal

Frontiers in Microbiology

Received: 25 February 2018

Accepted: 25 April 2018

Published: 14 May 2018

Citation:

Nan H, Lan J, Tian M, Dong S, Tian J, Liu L, XU X and Chen H (2018) The Network of Interactions

Among Porcine Reproductive and Respiratory Syndrome Virus

Non-structural Proteins.

Front. Microbiol. 9:970

doi: 10.3389/fmicb.2018.00970

\section{The Network of Interactions Among Porcine Reproductive and Respiratory Syndrome Virus Non-structural Proteins}

\author{
Hao Nan ${ }^{1}$, Jixun Lan ${ }^{1}$, Mengmeng Tian', Shan Dong ${ }^{1}$, Jiao Tian', Long Liu',2, \\ Xiaodong $\mathrm{Xu}^{1}$ and Hongying Chen ${ }^{1,3 *}$ \\ ${ }^{1}$ College of Life Sciences, Northwest A\&F University, Yangling, China, ${ }^{2}$ School of Basic Medical Sciences, Hubei University \\ of Medicine, Shiyan, China, ${ }^{3}$ Shaanxi Key Laboratory of Agricultural and Environmental Microbiology, College of Life \\ Sciences, Northwest A\&F University, Yangling, China
}

The RNA synthesis of porcine reproductive and respiratory syndrome virus (PRRSV), a positive-strand RNA virus, is compartmentalized in virus-induced double-membrane vesicles where viral proteins and some cellular proteins assemble into replication and transcription complexes (RTCs). The viral replicase proteins are the major components of the RTCs but the physical associations among these non-structural proteins (nsps) remain elusive. In this study, we investigated the potential interactions between PRRSV nsps by yeast two-hybrid (Y2H), bimolecular fluorescence complementation (BiFC) and pull-down assays. Our analyses revealed a complex network of interactions involving most of PRRSV nsps. Among them, nsp9 and nsp12 were identified as the hubs of the nsp interactome; transmembrane proteins nsp2 and nsp5 both interacted with nsp3, indicating that the three membrane-bound proteins might bind together to form the scaffold to support the association of RTCs with the intracellular membrane. The PRRSV nsp interactions identified in this study may provide valuable clues for future researches on the RTC formation and function.

\footnotetext{
Keywords: porcine reproductive and respiratory syndrome virus, non-structural proteins, protein-protein interaction, yeast two-hybrid, bimolecular fluorescence complementation assay, pull-down assay
}

\section{INTRODUCTION}

Porcine reproductive and respiratory syndrome virus (PRRSV), the causative agent of porcine reproductive and respiratory syndrome (PRRS) which has caused great economic losses to the pig industry, belongs to the family Arteriviridae in the order Nidovirales. It is an enveloped virus encapsulating a positive-stranded RNA genome of about $15 \mathrm{~kb}$. The genome contains at least 11 open reading frames (Fang et al., 2012; Kappes and Faaberg, 2015; Li et al., 2015). ORF1a and ORF1b at the 5'-proximal three quarters of the genome encode long polyproteins ppla and pplab which are subsequently cleaved by viral proteases to generate functional non-structural proteins (nsps), and the rest $3^{\prime}$ - proximal part of the genome consists of ORFs encoding viral structural proteins. A short alternative ORF that overlaps ORFla in the $+1 /-2$ frame was recently identified, and the ORF could be expressed to yield a transframe protein nsp2TF and a truncated nsp2 variant nsp2N by programmed ribosomal frameshifting (PRF) mechanism (Fang et al., 2012).

Among the nsps encoded by ORF1a, nspl $\alpha / \beta$ and nsp 2 contain papain-like proteinase (PLP) domains and are rapidly released by autoproteolytic cleavage of the polyproteins after their synthesis. nsp4 is the main proteinase that is responsible for the subsequent processing of 
the remaining polyproteins via a major processing pathway with nsp2 acting as a cofactor and a minor processing pathway in the absence of nsp2 (Li et al., 2015). Hydrophobic nsp2, nsp3, and nsp 5 containing predicted membrane-spanning domains are thought to be involved in membrane modification and formation of double membrane vesicles (DMVs), a typical structure induced by arterivirus infection. Nsp6 and nsp 8 are short peptides containing only 16 and 45 amino acid residues, respectively, and their functions in virus infection remain unknown. With an internal cleavage site, nsp7 can be further cleaved into nsp $7 \alpha$ and nsp7 $\beta$, however, only cleaved nsp7 $\alpha$ was detectable in PRRSV infected cells by western blot and radioimmunoprecipitation ( $\mathrm{Li}$ et al., 2012; Chen et al., 2017). The specific function of nsp7 is also unclear although structure-based reverse genetics studies have demonstrated that it plays an important role in arterivirus RNA synthesis (Manolaridis et al., 2011; Zhang et al., 2013).

ORF1b is the most conserved region of arterivirus genomes. It encodes four more nsps including the viral RNA dependent RNA-polymerase (RdRp) nsp9, RNA helicase nsp10, endoribonuclease (NendoU) nsp11 and nsp12 with unknown function (Fang and Snijder, 2010). As the key enzyme for viral RNA synthesis, nsp9 is considered to be a core component of the viral replication and transcription complex (RTC) (Knoops et al., 2012). The RdRp domain locates in the C-terminal part of nsp9, and the N-terminal part of the protein is identified as a nidovirus RdRp-associated nucleotidyltransferase domain (Lehmann et al., 2015).

During arterivirus infection, most nsps have been found to localize to membranes in the perinuclear region of the infected cell and assemble into a membrane-associated viral RTC (Pedersen et al., 1999; Knoops et al., 2012). The transmembrane proteins nsp2, nsp3, and nsp5 are believed to anchor the RTC to intracellular membranes and transform them into DMVs. Studies have found that arterivirus nsp2 interacts with nsp3 and the expression of the two nsps induces the formation of structures similar to the infection-induced DMVs (Snijder et al., 2001). PRRSV nsp $1 \alpha, n s p 1 \beta$ have also been reported to directly interact to nsp2 (Song et al., 2016). Our previous study have identified the binding of $n s p 7 \alpha$ to the viral RdRp (Chen et al., 2017). By immunofluorescence analysis, nsp $1 \beta$, nsp $2, \mathrm{nsp} 4, \mathrm{nsp} 7 \alpha, \mathrm{nsp} 7 \beta$, and nsp 8 have been observed to co-localize in distinct punctate foci in the perinuclear region of the cell, which is the site of viral RNA synthesis during the early stages of infection ( $\mathrm{Li}$ et al., 2012). However, how these nsps are associated and involved in the formation of RTC in DMVs remains to be elucidated.

In this study, we investigated the interactions among the PRRSV non-structural proteins by yeast two-hybrid (Y2H) and bimolecular fluorescence complementation (BiFC) assays, and verified some of the identified interactions by pull-down assays. We observed a complex network of interactions involving most of PRRSV nsps, which may provide a framework for future research on the RTC formation and function.

\section{MATERIALS AND METHODS}

\section{Virus Genes and Sequences}

The replicon FL-12 (Truong et al., 2004) of PRRSV North American strain NSVL 97-7895 (GenBank accessionno.AY545985.1) was used as the template for the amplification of nsp genes by PCR. The nsps used in this study and their known or predicted functions are listed in Table $\mathbf{1 .}$

\section{Yeast Two-Hybrid (Y2H) Screening of Nsp Interactions}

Each of the NSP gene segments was produced by polymerase chain reaction (PCR) using the corresponding primers listed in Supplementary Table S1, and then inserted into pGBKT7 and

\begin{tabular}{|c|c|c|}
\hline Name & $\mathrm{N}$ - and C-terminal residues of each polypeptide used in this study & Known or predicted functions \\
\hline NSP1 $\alpha$ & Met1-Met180 (180 aa) & $\begin{array}{l}\text { Accessory protease Papain-like cysteine protein protease- } \alpha \text {; viral } \\
\text { transcription factor; potential IFN antagonist }\end{array}$ \\
\hline NSP1 $\beta$ & Ala181-Gly383 (203 aa) & $\begin{array}{l}\text { Accessory protease Papain-like cysteine protein protease- } \beta \text {; potential } \\
\text { IFN antagonist }\end{array}$ \\
\hline NSP2 & $\begin{array}{l}\text { Ala384-Gly1579 (1196 aa) (for BiFC) } \\
\text { Ala384-Pro1197(814aa) (for Y2H) }\end{array}$ & $\begin{array}{l}\text { Accessory cysteine protease; transmembrane protein involved in } \\
\text { membrane modification; a member of the ovarian tumor domain (OTU) } \\
\text { family of deubiquitinating enzymes; potential IFN antagonist }\end{array}$ \\
\hline NSP3 & $\begin{array}{l}\text { Gly1580-Glu1809 (230 aa) (for BiFC) Try1602-Val1652 } \\
\text { (51aa) + Arg1749-Glu1809 (61aa) (for Y2H and pull-down ) }\end{array}$ & Transmembrane protein involved in membrane modification \\
\hline NSP4 & Gly1810-Glu2013 (204 aa) & A 3C-like serine proteases; potential IFN antagonist; apoptosis inducer \\
\hline NSP5 & $\begin{array}{l}\text { Gly2014-Glu2183 (170 aa) (for BiFC) } \\
\text { Gly2033-Arg2091(59aa) + Ala2111-Arg2131(21aa) (for Y2H and pull-down ) }\end{array}$ & $\begin{array}{l}\text { Transmembrane protein involved in membrane modification; suppressor } \\
\text { of JAK/STAT3 pathway }\end{array}$ \\
\hline NSP7 $\alpha$ & Ser2200-Glu2348 (149 aa) & Unknown \\
\hline NSP7 $\beta$ & Asn2349-Glu2458 (110 aa) & Unknown \\
\hline NSP8 & Ala2459-Cys2503 (45 aa) & Unknown \\
\hline NSP9 & Ala2459-Glu3143 (685 aa) & RNA-dependent RNA polymerase \\
\hline NSP10 & Gly3144-Glu3584 (441 aa) & NTPase; RNA helicase \\
\hline NSP11 & Gly3585-Glu3807 (223 aa) & Endoribonuclease (NendoU); potential IFN antagonist \\
\hline NSP12 & Gly3808-Asn3960 (153 aa) & Potential IFN inhibitor; inducer of proinflammatory cytokines \\
\hline
\end{tabular}


pGADT7, respectively. For transmembrane proteins nsp2, nsp3 and nsp5, the transmembrane domains identified by TMHMM Server v. 2.0 were deleted from the genes by PCR. The inserts in the constructs were verified by DNA sequencing.

The pGADT7-nsp and pGBKT7-nsp constructs were co-transformed in pairs into Saccharomyces cerevisiae (yeast) strain Y2HGold using the Yeastmaker Yeast Transformation System kit (Clontech, United States) according to the manufacturer's instruction. Selection of co-transformed colonies was performed on selective medium lacking leucine and tryptophan. Protein interactions were examined by growing on minimal synthetic medium lacking adenine, histidine, leucine, and tryptophan (SD/-Ade/-His/-Leu/-Trp).

\section{Bimolecular Fluorescence Complementation (BiFC) Assay}

The full-length nsp gene fragments used in BiFC assays (listed in Table 1) were amplified by PCR using the corresponding primers listed in Supplementary Table S1, and then, respectively, inserted into vectors pBiFC-VN173 or pBiFC-VC155 (Addgene) for the expression of fusion proteins nsp-YFPN and nsp-YFPC. Human embryonic kidney (HEK) 293T cells were co-transfected in pairs with the constructs using calcium phosphate. Cells co-transfected with pBiFC-bjun-VN173 and pBiFC-bFosVC155 were used as positive control, and cells co-transfected with pBiFC-bjun-VN173 and pBiFC-bFos(deltaZIP)VC155 were set as negative control. The supernatant was replaced with fresh medium at $8 \mathrm{~h}$ post-transfection, and the fluorescence was examined $24 \mathrm{~h}$ post-transfection using a DM5000B microscope (Leica, Germany).

\section{Protein Expression in Escherichia coli and Purification}

The expression and purification of nsp9-His (Liu et al., 2016), His-nsp $7 \alpha$ and His-nsp7 $\beta$ (Chen et al., 2017) have been described previously. His-tagged tobacco etch virus protease (TEV-His) (Chen et al., 2017) was purified and used as a negative control. To express GST-nsp3 and GST-nsp5, the nsp genes without the transmembrane regions were amplified by over-lapping PCR and cloned into pGEX-2T vector. The exact expressed regions for each nsp are illustrated in Table 1. The GST-tagged proteins expressed in Escherichia coli BL21(DE3) strain were purified with GST-Bind Sepharose (CWBIO) in phosphate buffer $(137 \mathrm{mM}$ $\mathrm{NaCl}, 2.7 \mathrm{mM} \mathrm{KCl}, 10 \mathrm{mM} \mathrm{Na} \mathrm{HPO}_{4}$, and $2 \mathrm{mM} \mathrm{KH} \mathrm{PO}_{4}$ [pH 8.0]).

The nsp12 gene was amplified by PCR and inserted into vector pMAL-c4X (New England Biolabs). MBP-nsp12 was expressed in E. coli and purified using Amylose Resin (New England Biolabs) according to the manufacturer's instruction.

To express Flag-tagged proteins, a pTriEx-flag vector was made by inserting annealed oligonucleotides Nco-flag ( $5^{\prime}$ CATGGATTACAAGGATGACGATGACAAGG-3') and Bamflag (5'-GATCCCTTGTCATCGTCATCCTTGTAATC-3') into pTriEx1.1 (Novagene) between the NcoI and BamHI sites. The nsp $1 \alpha$, nsp $7 \alpha$, nsp 8 and nsp 11 genes were amplified by PCR and cloned into pTriEx-flag for the expression of Flag-tagged nsps.

\section{Pull-Down Assay}

His-tagged protein was mixed with GST/Flag tagged nsp. The mixture was incubated at $4^{\circ} \mathrm{C}$ for $2 \mathrm{~h}$ and mixed with PureProteome nickel magnetic beads (Millipore, United States). Proteins captured by the beads were eluted with elution buffer (50 mM sodium phosphate, $300 \mathrm{mM} \mathrm{NaCl}$, and $300 \mathrm{mM}$ imidazole [ $\mathrm{pH} 8.0]$ ), and then separated by SDS-PAGE and detected by Western blotting using tag antibodies (CWBIO). Similarly, MBP-nsp12 was mixed with GST/Flag/His tagged nsps, and the proteins co-purified with MBP-nsp12 using Amylose Resin were then separated by SDS-PAGE, stained with Coomassie brilliant blue or detected by Western blotting.

\section{RESULTS}

\section{Screening Nsp Interactions by Yeast Two-Hybrid}

After the replicase polyprotein translation and processing in PRRSV infection, most of the non-structural proteins are believed to be involved in the RTC formation and participate in the virus replication and transcription processes. In order to investigate the associations between PRRSV nsps, we performed a yeast two-hybrid matrix screen with all the viral nsps except nsp6, which is only 16 amino acids in length. As both the $\mathrm{AD}$ - and $\mathrm{BD}$-fused proteins need to enter the nucleus to activate the reporter expression, the putative transmembrane domains in nsp2, nsp3, and nsp5 were deleted to avoid detaining the transmembrane proteins with intracellular membranes (the peptides used in the study is listed in Table 1). The gene fragments of the tested nsps were cloned into both the pGBKT7 and pGADT7 vectors so that they could be tested as both prey and bait.

The yeast two-hybrid screening resulted in 30 positive results out of the 169 tested protein combinations (Figure 1 and Supplementary Figure S1), including previously reported interaction pairs of $n s p 1 \alpha-n s p 1 \alpha$ (Sun et al., 2009), nsp $1 \beta-n s p 1 \beta$ (Xue et al., 2010), nsp2-nsp3 (Snijder et al., 2001), nsp7 $\alpha-n s p 9$ (Chen et al., 2017), and nsp11-nsp11 (Shi et al., 2016). Among the positive interactions, 7 protein pairs (nsp2-nsp3, nsp3-nsp5, nsp3-nsp12, nsp5-nsp12, nsp7 $\alpha$-nsp12, nsp7 $\beta$-nsp9, and nsp7 $\beta$-nsp12) gave positive results no matter which one served as the bait or prey. Protein self-interactions were identified in 6 nsps (nsp1 $\alpha$, nsp1 $\beta$, nsp3, nsp5, nsp11, and nsp12). Although only the interaction with nsp7 $\beta$ was detected for nsp 9 as the prey, nsp9 fused with GAL4 BD domain was found to interact with 7 prey nsps (nsp1 $\alpha, n s p 3, n s p 7 \alpha, n s p 7 \beta, n s p 8, n s p 11$, and nsp12), supporting it as a prospective core component of the viral RTC. Nsp5 interacted with $n s p 7 \alpha$ and nsp7 $\beta$ only when it served as the prey. The interactions of nsp10 with nsp3 and nsp12 were positive when nsp10 acted as the bait. In $\mathrm{Y} 2 \mathrm{H}$, the bait is fused to the DNA binding domain and the prey is fused to the activation domain of the transcription factor. The different fusion partner and the relative position to the DNA strand may affect the folding and accessibility of the fusion proteins, which may result in the discrepancy in the interaction results for the nsps, 
Fused with GAL4 DNA-Binding Domain (BD)

\begin{tabular}{|c|c|c|c|c|c|c|c|c|c|c|c|c|c|}
\hline & nsp1 $1 \alpha$ & nsp1 $\beta$ & nsp2 & nsp3 & nsp4 & nsp5 & nsp7 $\alpha$ & nsp7 $\beta$ & nsp8 & nsp9 & nsp10 & nsp11 & nsp12 \\
\hline nsp1 $\alpha$ & + & - & - & - & - & - & - & - & - & + & - & - & - \\
\hline nsp1 $\beta$ & - & + & - & - & - & - & - & - & - & - & - & - & - \\
\hline nsp2 & - & - & - & + & - & - & - & - & - & - & - & - & - \\
\hline nsp3 & - & - & + & + & - & + & - & - & - & + & + & - & + \\
\hline nsp4 & - & - & - & - & $=$ & - & - & - & - & - & - & - & - \\
\hline nsp5 & - & - & - & + & - & + & + & + & - & - & - & - & + \\
\hline nsp7 $\alpha$ & - & - & - & - & - & - & - & - & - & + & - & - & + \\
\hline nsp7 $\beta$ & - & - & - & - & - & - & - & - & - & + & - & - & + \\
\hline nsp8 & - & - & - & - & - & - & - & - & - & + & - & - & - \\
\hline nsp9 & - & - & - & - & - & - & - & + & - & $=$ & - & - & - \\
\hline nsp10 & - & - & - & - & - & - & - & - & - & - & - & - & - \\
\hline nsp11 & - & - & - & - & - & - & - & - & - & + & - & + & - \\
\hline nsp12 & - & - & - & + & - & + & + & + & - & + & + & - & + \\
\hline
\end{tabular}

FIGURE 1 | Analysis of PRRSV non-structural protein interactions by Y2H matrix screen. +, positive interaction; -, negative interaction.

respectively, acting as a bait or a prey. No positive interaction was identified for the main proteinase nsp4, whose associations with the replicase polypeptides might be very short-lived.

\section{BiFC Analysis of Nsp Interactions}

To eliminate potential false positive interactions between nsps identified by $\mathrm{Y} 2 \mathrm{H}$, the non-structural proteins were, respectively, fused to the $\mathrm{N}$ - or C-terminal part of enhanced yellow fluorescent protein (YFP), and protein-protein interactions were visualized under a fluorescence microscope. As shown in Figure 2, most of the combinations of nsps showed positive interactions in $\mathrm{Y} 2 \mathrm{H}$ were confirmed in the BiFC assay, with the exception of nsp3-nsp10 (Figure 2C) and nsp5-nsp5 (Figure 2D) which did not yield observable fluorescence. Considering that the full-length transmembrane proteins were used in $\mathrm{BiFC}$ analysis but the transmembrane domains were deleted in $\mathrm{Y} 2 \mathrm{H}$ assays, this result implied that the nsp5-nsp5 and nsp3-nsp10 interactions identified in $\mathrm{Y} 2 \mathrm{H}$ using truncated nsp5 and nsp3 might be artifacts.

On the other hand, we observed three more interactions (nsp1 $\beta$-nsp9, nsp8-nsp12, and nsp11-nsp12) in BiFC assays which were not detected by $\mathrm{Y} 2 \mathrm{H}$. BiFC enables direct visualization of protein interactions in living cells. It has been reported that it can detect weak interactions and the fluorophore reconstitution can occur at intermolecular distances over $7 \mathrm{~nm}$
(Hu et al., 2006; Kerppola, 2008). This may contribute to less steric hindrance and higher sensitivity in the detection of protein-protein interaction. In addition, $\mathrm{Y} 2 \mathrm{H}$ takes place in the nucleus. For most PRRSV nsps which normally only localize in the cytoplasm, proteins detected interacting in the cytoplasm may be found to be non-interacting in the nucleus in $\mathrm{Y} 2 \mathrm{H}$.

Interacted nsp2-nsp3 and nsp3-nsp5 appeared as punctate foci in the perinuclear region of the cell, similar to the distribution of nsps observed in early infection (Li et al., 2012) and consistent with previous observations that expression of these transmembrane nsps could induce the formation of structures similar to the infection-induced DMVs (Snijder et al., 2001; van der Hoeven et al., 2016).

\section{Verification of Nsp Interactions by Pull-Down Assay}

By $\mathrm{Y} 2 \mathrm{H}$ and BiFC, multiple interaction partners were detected for nsp 9 , nsp12 and transmembrane proteins nsp3 and nsp5, suggesting that these nsps might act as the core components in RTC formation. Pull-down assays were then performed to further validate these multiple interactions. Using nsp9-His as bait, the binding of nsp1 $\alpha, n s p 3, n s p 7 \alpha$ and nsp11 to nsp9 were confirmed, but the nsp8-nsp9 interaction was not detected (Figure 3A). We observed that nsp8 was sensitive to degradation, therefore, the rapid degradation of the protein during the 


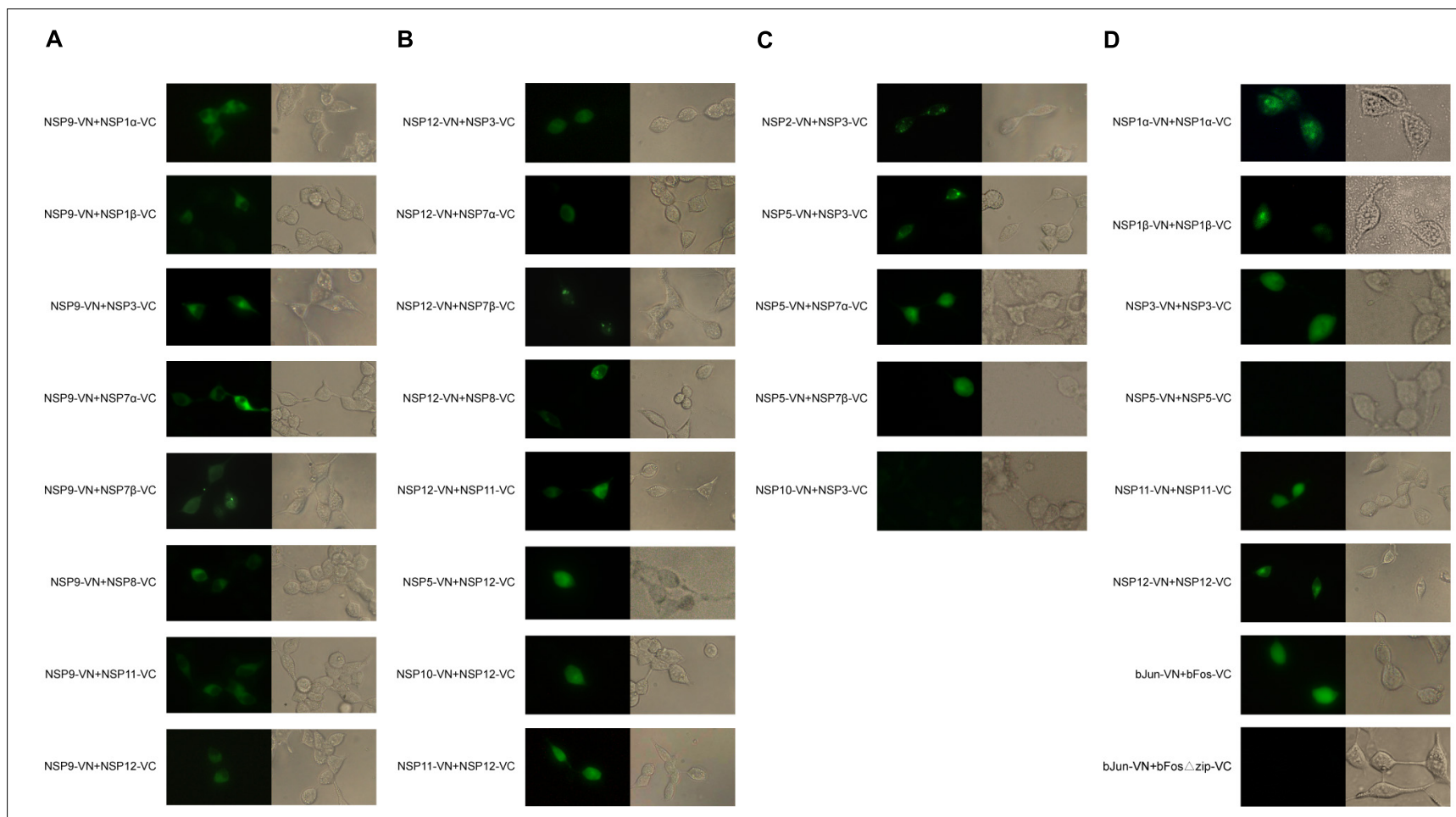

FIGURE 2 | BiFC analysis of nsp interactions. Each nsp was fused to either the N- or C-terminal part of YFP. The fusion proteins were expressed in HEK 293T cells and YFP images in cells were visualized at $24 \mathrm{~h}$ post-transfection. (A) Interactions between nsp9 and other nsps. (B) Interactions between nsp12 and other nsps. (C) Interactions involving transmenbrane nsps. (D) Self-interactions and controls. Protein pair bJun-VN + bFos-VC was used as positive control, and bJun-VN + bFos(delta)zip-VC was negative control.

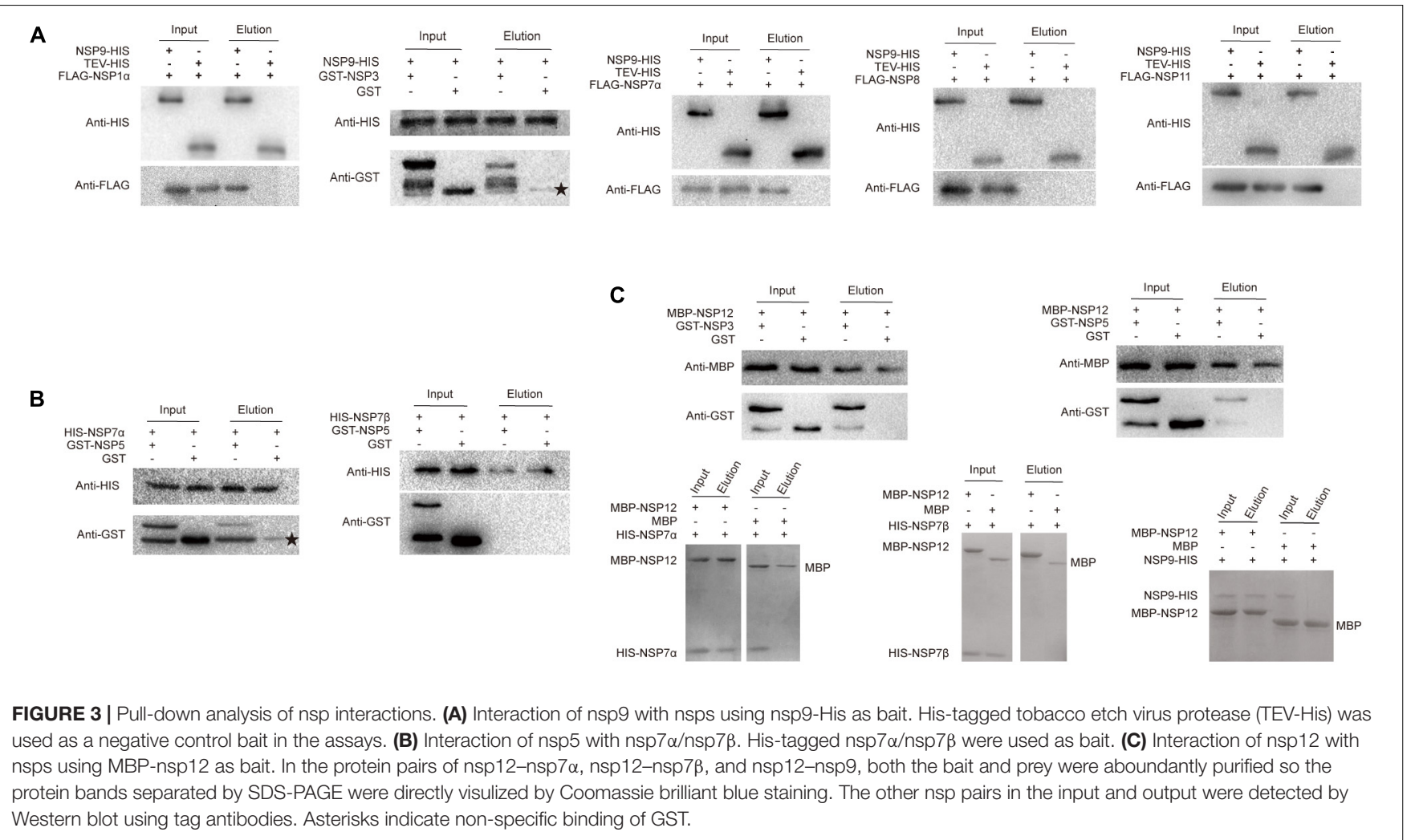




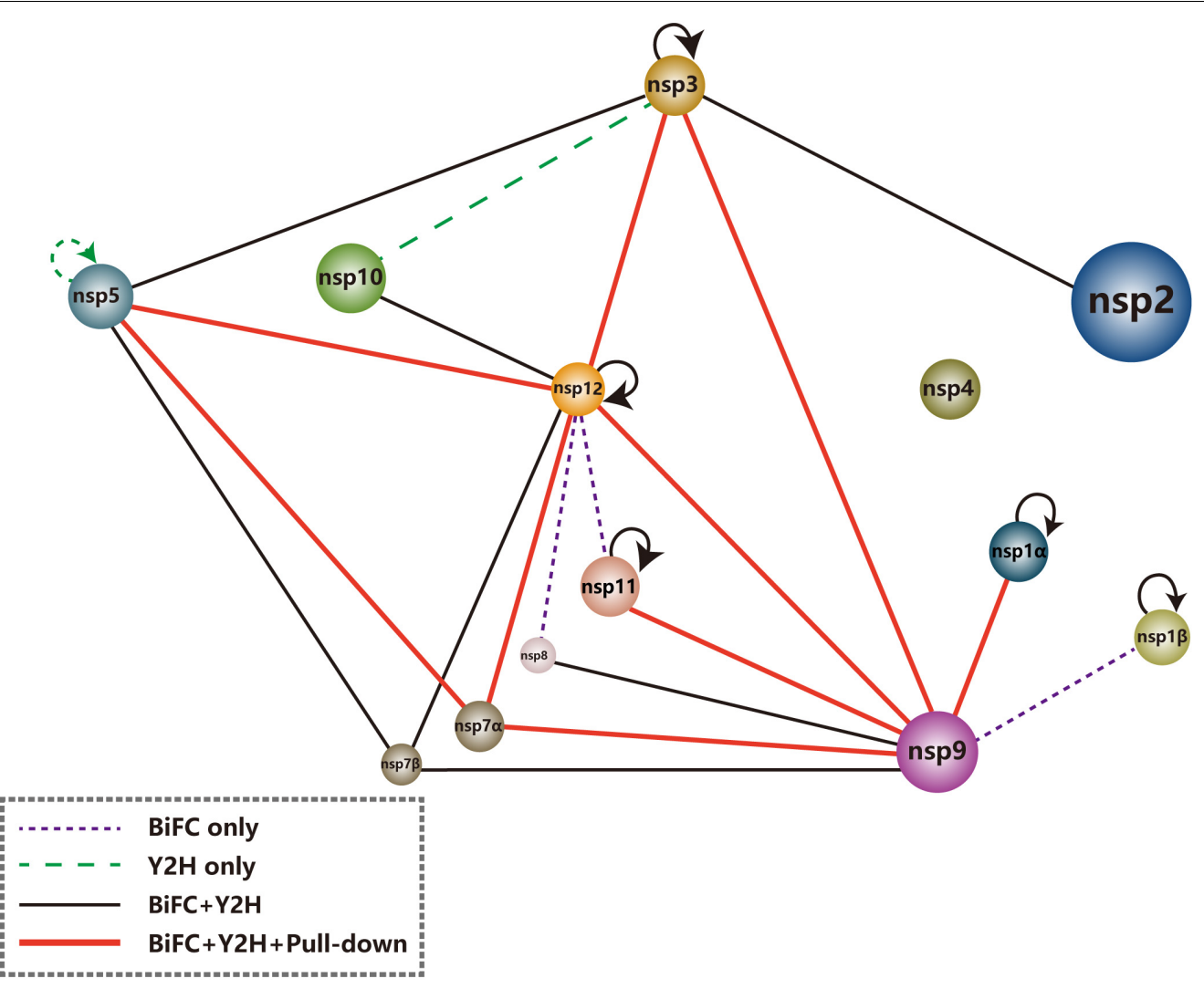

FIGURE 4 | Schematic summary of nsp interactions identified by $Y 2 \mathrm{H}, \mathrm{BiFC}$, and pull-down assays.

incubation of protein mixture could probably contribute to the failure in the detection of eluted nsp8 in pull-down assay.

To express the transmembrane proteins nsp3 and nsp5 in E. coli, the predicted hydrophobic transmembrane domains located in the middle of the polypeptides were deleted and the hydrophilic regions were linked by a GGGGS $\times 2$ linker. The proteins were fused to both His-tag and GST-tag, however, the His-tagged proteins were predominantly expressed as insoluble inclusion bodies (data not shown) and only the GST-tagged nsp3 and nsp5 were moderately soluble and suit for pull-down assay. GST-nsp3 and GST-nsp5 were used as prey to detect their interactions with His-tagged nsp $7 \alpha$, nsp7 $\beta$, nsp9, and MBP-tagged nsp12. Although a faint GST band as the control prey was sometimes observed to bind to the nickel magnetic beads (indicated by asterisks in Figures 3A,B) and cleaved GST was consistently detected in all the samples containing GST-nsp3/nsp5, the binding of GST-nsp3 to nsp9-his (Figure 3A) and GST-nsp5 to His-nsp7 $\alpha$ (Figure 3B) was obvious. The interaction of GST-nsp5 to His-nsp7 $\beta$ was not detected (Figure 3B), probably due to the instability characteristics of nsp7 $\beta$ that was previously reported (Li et al., 2012) and also observed in our studies.

For pull-down analysis of the interaction of nsp12 with other nsps, MBP-tagged nsp12 was purified and used as bait. As shown in Figure 3C, the interactions of nsp12 with nsp3, nsp5, nsp7 $\alpha$, and nsp9 were clearly observed. Similar to the negative result for the interaction between GST-nsp5 and His-nsp7 $\beta$, no His-nsp7 $\beta$ was detected binding to MBP-nsp12.

\section{PRRSV Nsp Interactome}

Based on the $\mathrm{Y} 2 \mathrm{H}, \mathrm{BiFC}$ and pull-down results in this study, protein-protein interactions among PRRSV nsps are summarized in Figure 4. In this network, the viral RdRp (nsp9) was found to associate with multiple interaction partners including $\mathrm{nsp} 1 \alpha, \mathrm{nsp} 1 \beta$, nsp3, nsp $7 \alpha, \mathrm{nsp} 7 \beta$, nsp8, nsp11, and nsp12. Most of these interactions were screened by $\mathrm{Y} 2 \mathrm{H}$ and verified both by BiFC and pull-down assays, except that the interaction with nsp $1 \beta$ was identified by BiFC and the associations with nsp7 $\beta$ and nsp8 were not detected by pull-down. Nsp7 $\beta$ and nsp8 were easily degradable and they were probably degraded during the in vitro assay. The multiple nsp associations of nsp9 validated the speculation of the RdRp as a core component of the viral RTC.

The second hub identified in the PRRSV nsp interactome was nsp 12 , whose interactions with nsp3, nsp5, nsp7 $\alpha$, and nsp9 were detected by all the three assays used in this study. The interactions of nsp12 with nsp7 $\beta$, nsp10 and nsp12 itself were determined by both $\mathrm{Y} 2 \mathrm{H}$ and BiFC. The nsp8-nsp12 and nsp11-nsp12 associations were only observed by BiFC.

Both transmembrane protein $\mathrm{nsp} 2$ and $\mathrm{nsp} 5$ interacted with transmembrane protein nsp3, and nsp3 could also bind to itself, 


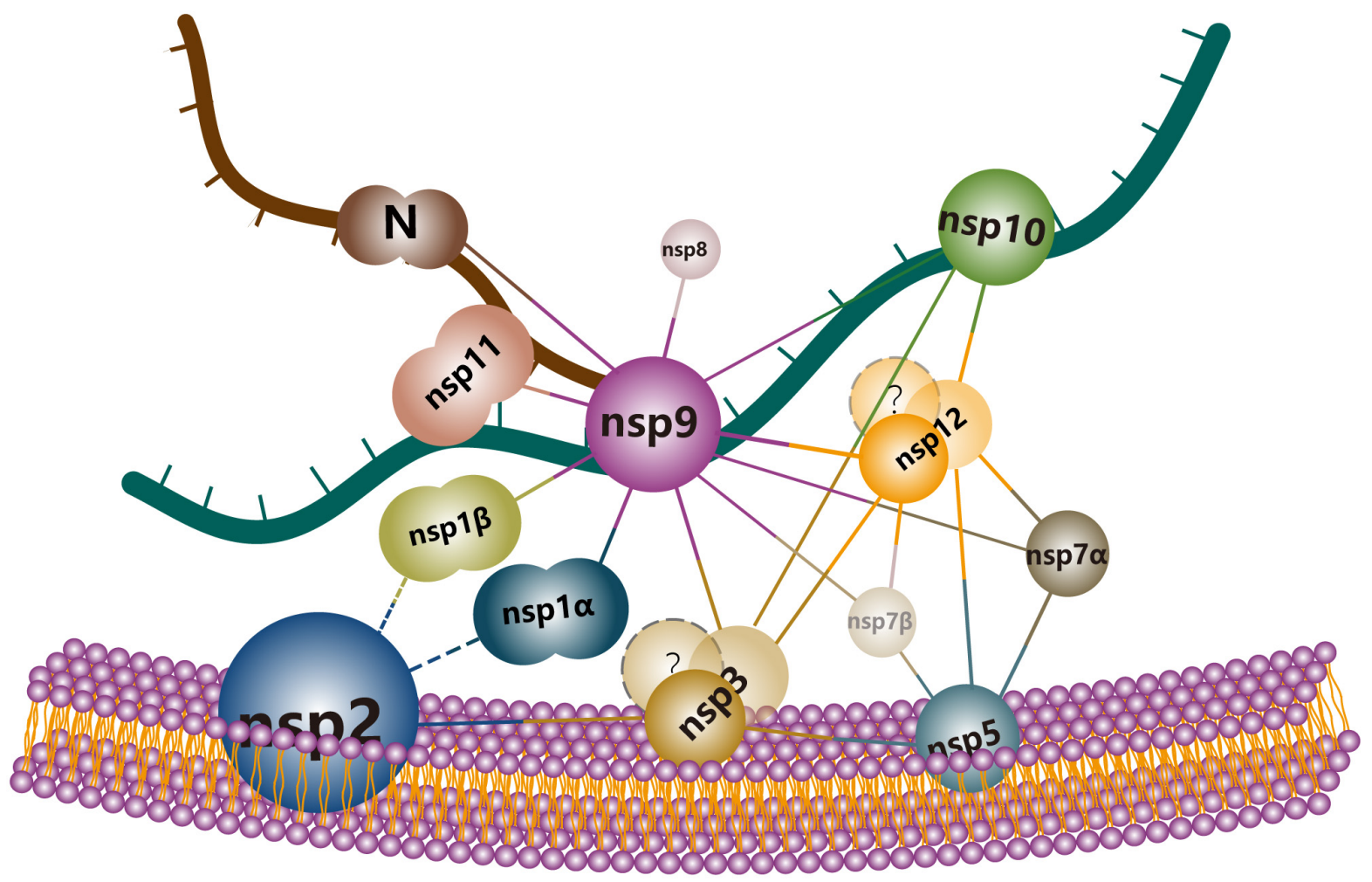

FIGURE 5 | A model of PRRSV replication and transcription complex in DMVs. The three PRRSV transmembrane proteins (nsp2, nsp3, and nsp5) form a membrane-bound nsp2-nsp3(xn)-nsp5 scaffold for the support of other nsp components. Amongst the soluble nsps, nsp9 and nsp12 act as hubs in the nsp interaction network by interacting with multiple partners including both transmemebrane and soluble nsps. The interactions identified in our assays are linked with solid lines and the documented associations of nsp2 with nsp1 $\alpha$ and nsp1 $\beta$ which were not detected in this study were indicated by dashed lines.

suggesting that these three nsps might associate together to form a membrane-bound complex. Through the direct interactions of nsp3 with soluble proteins nsp9, nsp12 and maybe also nsp10, and the direct binding of nsp5 to $n s p 7 \alpha, n s p 7 \beta$, and nsp12, the transmembrane proteins indirectly associated with most other nsps. As no interaction of nsp4 with other nsps was observed by $\mathrm{Y} 2 \mathrm{H}$, we did not further study it in BiFC and pull-down assays.

\section{DISCUSSION}

Like other positive-stranded RNA viruses, the replication and transcription of arteriviruses is compartmentalized by double-membrane vesicles (DMVs) (Pedersen et al., 1999; Snijder et al., 2001), where viral and some cellular proteins (van Hemert et al., 2008; Beura et al., 2011) form replication and transcription complexes (RTCs). The viral non-structural proteins are the major components of the RTCs and the transcription and replication of arteriviruses have been extensively studied, but the physical relationships among the viral proteins required for this process are mainly unknown. In this study, we investigated the network of interactions amongst the non-structural proteins that assemble into the PRRSV RTC.
It has long been known that two transmembrane non-structural proteins (nsp2 and nsp3) of arterivirus equine arteritis virus (Fuchs et al., 1983) interact with each other and they are suffice to induce DMVs comparable to those formed during viral infection (Snijder et al., 2001). Recently, it was found that coexpression of nsp 5 could result in the formation of more homogenous DMVs that were closer to what was observed upon EAV infection, suggesting that nsp5 played a regulatory role in modulating membrane curvature and DMV formation (van der Hoeven et al., 2016). Belonging to the same family Arterivirus, PRRSV has similar gene structure and closely related to EAV. It is conceivable that PRRSV transmembrane proteins nsp2, nsp3, and nsp5 may also recognize each other and act as the same roles in the DMV formation. By $\mathrm{Y} 2 \mathrm{H}$ and $\mathrm{BiFC}$, we found that both nsp2 and nsp5 interacted with nsp3, and nsp3 could also interact itself, indicating that the three PRRSV transmembrane proteins might form a membrane-bound nsp2-nsp3 $(\times n)$-nsp5 scaffold to support the formation of RTC for the viral replication and transcription (a model is illustrated in Figure 5). This model is consistent with previous finding that EAV nsp3 plays a key role in the remodeling of intracellular membranes (Posthuma et al., 2008). It is unclear if EAV nsp3 can also bind to itself, and further studies are needed to investigate whether PRRSV nsp3 is anchored on the intracellular membrane as a dimer or higher multimer. 
As the viral RNA polymerase, arterivirus nsp9 has been thought as a core component in RTCs. Although previous studies have found that PRRSV nsp9 can interact with viral structural protein $\mathrm{N}$ (Liu et al., 2016), viral non-structural protein nsp7 $\alpha$ (Chen et al., 2017), and some cellular proteins such as retinoblastoma protein (Dong et al., 2014) and DEAD-box RNA helicase 5 (Zhao et al., 2015), how nsp9 associates with other nsps is not understood. Here, we confirmed the nsp $7 \alpha-n s p 9$ interaction and detected the direct binding of seven more nsps (nsp1 $\alpha, \mathrm{nsp} 1 \beta$, nsp3, nsp7 $\beta$, nsp8, nsp11, and nsp12) to nsp9. These data highlighted the central role of nsp9 within the RTC machinery.

During the infection of nidoviruses including arteriviruses and coronaviruses, the viruses produce the full-length genome using continuously synthesized minus-stranded RNA as template. They also generate a nested set of subgenomic mRNAs by a unique mechanism of discontinuous minus-strand RNA template synthesis (Sawicki and Sawicki, 1995). The relative abundance of the genomic and subgenomic RNAs is well controlled in the virus infection, and this is vital for efficient virus production (Nedialkova et al., 2010). It is unclear how the RTC fine-tunes these various RNA synthesis processes. The association of specific replicase subunits with the RNA polymerase may regulate the balance between replication and transcription. EAV nsp1 has been identified as a "transcription factor" for the RdRp switch from continuous into discontinuous mode (Nedialkova et al., 2010). Our interactome analyses detected the direct binding of nsp $1 \alpha$ to nsp9 by all the three assays, consisting with the previous observation that the $\mathrm{N}$-terminal zinc finger domain of nsp1 was important for this function (Tijms et al., 2001; Nedialkova et al., 2010). Mutations in other nspl domains were also found to strongly influence transcription (Tijms et al., 2001; Nedialkova et al., 2010). We did not detect the interaction of $n s p 1 \beta$ to any nsps other than itself in $\mathrm{Y} 2 \mathrm{H}$ screening, but nsp $1 \beta-n s p 9$ interaction was clearly observed by BiFC. It has been observed that $n s p 1 \alpha$ and nsp $1 \beta$ also directly interact with nsp2 by immunoprecipitation (Song et al., 2016). Our Y2H assay showed negative results for these two pairs, which might be false negative results due to the relatively large sizes of the protein pairs, and further studies are needed to explore this.

An interactome study of SARS-coronavirus nsps demonstrated that viral key enzymes encoded by ORF1b, including the RNA helicase (nsp13), 3'-5' exonuclease (nsp14), endonuclease (nsp15) and putative methyltransferase (nsp16), all bound to the RdRp (nsp12), leading to the hypothesis of the existence of a proofreading mechanism for the faithful replication and transcription of the viral long RNA genome (Imbert et al., 2008). For PRRSV, we observed the interaction of the endonuclease (nsp11) to the RdRp (nsp9), but did not detect the direct association of the RNA helicase (nsp10) with nsp9.

\section{REFERENCES}

Beura, L. K., Dinh, P. X., Osorio, F. A., and Pattnaik, A. K. (2011). Cellular poly(c) binding proteins 1 and 2 interact with porcine reproductive and respiratory
However, our data revealed an indirect interaction of PRRSV nsp10 with nsp9 via nsp12 (Figure 4).

Besides nsp9, the protein-protein interaction analyses also identified multiple interaction partners (nsp3, nsp5, nsp7 $\alpha$, nsp7 $\beta$, nsp8, nsp9, nsp10, nsp11, and nsp12 itself) for nsp12, the structure and function of which is still unknown. Using high throughput proteomics, we have found that PRRSV nsp12 interacts with many cellular proteins with high probability (Dong et al., 2016). Among the cellular proteins, HSP70 is recruited by nsp 12 to maintain the protein's stability and benefit the viral replication. Another study have shown that nsp12 induces the phosphorylation of STAT1 at serine 727 and the expression of proinflammatory cytokines IL-1 $\beta$ and IL-8 (Yu et al., 2013). In a most recent report, nsp12 is documented to be able to induce the stabilization of karyopherin alpha6 which is required for the nuclear translocation of $n s p 1 \beta$ and the PRRSV replication (Yang et al., 2018). These previous data have demonstrated the involvement of PRRSV nsp12 in the virus infection by modulating cellular factors. The identification of multiple interactions of nsp12 with viral nsps in this study suggests that nsp12, which probably exists as a homologous dimer or multimer by self-interaction, should also play an important role in the viral RNA synthesis process as a crucial component of the RTC.

\section{CONCLUSION}

We investigated the potential interactions between PRRSV nsps, which may be involved in the formation of viral replication and transcription complex. Our data confirmed most of the documented interactions among PRRSV nsps, and identified a number of novel associations, which may provide valuable clues for future researches such as the viral transcription and replication mechanism and antiviral drug design.

\section{AUTHOR CONTRIBUTIONS}

HC and XX conceived and designed the research. HN, JL, MT, $\mathrm{SD}$, JT, and LL performed the $\mathrm{Y} 2 \mathrm{H}$ screening. HN, JL, and JT conducted the BiFC assays. HN and JL carried out pull-down assays. $\mathrm{HC}, \mathrm{XX}, \mathrm{HN}$, and $\mathrm{JL}$ analyzed the data. HC wrote the manuscript.

\section{SUPPLEMENTARY MATERIAL}

The Supplementary Material for this article can be found online at: https://www.frontiersin.org/articles/10.3389/fmicb. 2018.00970/full\#supplementary-material

syndrome virus nonstructural protein lbeta and support viral replication. J. Virol. 85, 12939-12949. doi: 10.1128/JVI.05177-11

Chen, J., Xu, X., Tao, H., Li, Y., Nan, H., Wang, Y., et al. (2017). Structural analysis of porcine reproductive and respiratory syndrome virus non-structural protein 
7alpha (NSP7alpha) and identification of its interaction with NSP9. Front. Microbiol. 8:853. doi: 10.3389/fmicb.2017.00853

Dong, J., Zhang, N., Ge, X., Zhou, L., Guo, X., and Yang, H. (2014). The interaction of nonstructural protein 9 with retinoblastoma protein benefits the replication of genotype 2 porcine reproductive and respiratory syndrome virus in vitro. Virology 464-465, 432-440. doi: 10.1016/j.virol.2014.07.036

Dong, S., Liu, L., Wu, W., Armstrong, S. D., Xia, D., Nan, H., et al. (2016). Determination of the interactome of non-structural protein 12 from highly pathogenic porcine reproductive and respiratory syndrome virus with host cellular proteins using high throughput proteomics and identification of HSP70 as a cellular factor for virus replication. J. Proteom. 146, 58-69. doi: 10.1016/j. jprot.2016.06.019

Fang, Y., and Snijder, E. J. (2010). The PRRSV replicase: exploring the multifunctionality of an intriguing set of nonstructural proteins. Virus Res. 154, 61-76. doi: 10.1016/j.virusres.2010.07.030

Fang, Y., Treffers, E. E., Li, Y., Tas, A., Sun, Z., van der Meer, Y., et al. (2012). Efficient -2 frameshifting by mammalian ribosomes to synthesize an additional arterivirus protein. Proc. Natl. Acad. Sci. U.S.A. 109, E2920-E2928. doi: 10.1073/ pnas. 1211145109

Fuchs, L. Y., Woods, M. S., and Weaver, R. F. (1983). Viral transcription during Autographa californica nuclear polyhedrosis virus infection: a novel RNA polymerase induced in infected Spodoptera frugiperda Cells. J. Virol. 48, 641-646.

Hu, C. D., Grinberg, A. V., and Kerppola, T. K. (2006). Visualization of protein interactions in living cells using bimolecular fluorescence complementation (BiFC) analysis. Curr. Protoc. Cell Biol. Chapter 21: Unit 21.23.

Imbert, I., Snijder, E. J., Dimitrova, M., Guillemot, J. C., Lecine, P., and Canard, B. (2008). The SARS-Coronavirus PLnc domain of nsp3 as a replication/transcription scaffolding protein. Virus Res. 133, 136-148. doi: 10.1016/j.virusres.2007.11.017

Kappes, M. A., and Faaberg, K. S. (2015). PRRSV structure, replication and recombination: Origin of phenotype and genotype diversity. Virology 479-480, 475-486. doi: 10.1016/j.virol.2015.02.012

Kerppola, T. K. (2008). Bimolecular fluorescence complementation (BiFC) analysis as a probe of protein interactions in living cells. Annu. Rev. Biophys. 37, 465-487. doi: 10.1146/annurev.biophys.37.032807.125842

Knoops, K., Barcena, M., Limpens, R. W., Koster, A. J., Mommaas, A. M., and Snijder, E. J. (2012). Ultrastructural characterization of arterivirus replication structures: reshaping the endoplasmic reticulum to accommodate viral RNA synthesis. J. Virol. 86, 2474-2487. doi: 10.1128/JVI.06677-11

Lehmann, K. C., Gulyaeva, A., Zevenhoven-Dobbe, J. C., Janssen, G. M., Ruben, M., Overkleeft, H. S., et al. (2015). Discovery of an essential nucleotidylating activity associated with a newly delineated conserved domain in the RNA polymerasecontaining protein of all nidoviruses. Nucleic Acids Res. 43, 8416-8434. doi: $10.1093 /$ nar/gkv838

Li, Y., Tas, A., Snijder, E. J., and Fang, Y. (2012). Identification of porcine reproductive and respiratory syndrome virus ORF1a-encoded non-structural proteins in virus-infected cells. J. Gen. Virol. 93, 829-839. doi: 10.1099/vir.0. 039289-0

Li, Y., Tas, A., Sun, Z., Snijder, E. J., and Fang, Y. (2015). Proteolytic processing of the porcine reproductive and respiratory syndrome virus replicase. Virus Res. 202, 48-59. doi: 10.1016/j.virusres.2014.12.027

Liu, L., Tian, J., Nan, H., Tian, M., Li, Y., Xu, X., et al. (2016). Porcine reproductive and respiratory syndrome virus nucleocapsid protein interacts with Nsp9 and cellular DHX9 to regulate viral RNA synthesis. J. Virol. 90, 5384-5398. doi: 10.1128/JVI.03216-15

Manolaridis, I., Gaudin, C., Posthuma, C. C., Zevenhoven-Dobbe, J. C., Imbert, I., Canard, B., et al. (2011). Structure and genetic analysis of the arterivirus nonstructural protein 7alpha. J. Virol. 85, 7449-7453. doi: 10.1128/JVI. 00255-11

Nedialkova, D. D., Gorbalenya, A. E., and Snijder, E. J. (2010). Arterivirus Nsp1 modulates the accumulation of minus-strand templates to control the relative abundance of viral mRNAs. PLoS Pathog. 6:e1000772. doi: 10.1371/journal.ppat. 1000772

Pedersen, K. W., van der Meer, Y., Roos, N., and Snijder, E. J. (1999). Open reading frame la-encoded subunits of the arterivirus replicase induce endoplasmic reticulum-derived double-membrane vesicles which carry the viral replication complex. J. Virol. 73, 2016-2026.
Posthuma, C. C., Pedersen, K. W., Lu, Z., Joosten, R. G., Roos, N., ZevenhovenDobbe, J. C., et al. (2008). Formation of the arterivirus replication/transcription complex: a key role for nonstructural protein 3 in the remodeling of intracellular membranes. J. Virol. 82, 4480-4491. doi: 10.1128/JVI.02756-07

Sawicki, S. G., and Sawicki, D. L. (1995). Coronaviruses use discontinuous extension for synthesis of subgenome-length negative strands. Adv. Exp. Med. Biol. 380, 499-506. doi: 10.1007/978-1-4615-1899-0_79

Shi, Y., Li, Y., Lei, Y., Ye, G., Shen, Z., Sun, L., et al. (2016). A dimerizationdependent mechanism drives the endoribonuclease function of porcine reproductive and respiratory syndrome virus nsp11. J. Virol. 90, 4579-4592. doi: 10.1128/JVI.03065-15

Snijder, E. J., van Tol, H., Roos, N., and Pedersen, K. W. (2001). Non-structural proteins 2 and 3 interact to modify host cell membranes during the formation of the arterivirus replication complex. J. Gen. Virol. 82, 985-994. doi: 10.1099/ 0022-1317-82-5-985

Song, T., Fang, L., Wang, D., Zhang, R., Zeng, S., An, K., et al. (2016). Quantitative interactome reveals that porcine reproductive and respiratory syndrome virus nonstructural protein 2 forms a complex with viral nucleocapsid protein and cellular vimentin. J. Proteom. 142, 70-81. doi: 10.1016/j.jprot.2016.05.009

Sun, Y., Xue, F., Guo, Y., Ma, M., Hao, N., Zhang, X. C., et al. (2009). Crystal structure of porcine reproductive and respiratory syndrome virus leader protease Nsplalpha. J. Virol. 83, 10931-10940. doi: 10.1128/JVI.02579-08

Tijms, M. A., van Dinten, L. C., Gorbalenya, A. E., and Snijder, E. J. (2001). A zinc finger-containing papain-like protease couples subgenomic mRNA synthesis to genome translation in a positive-stranded RNA virus. Proc. Natl. Acad. Sci. U.S.A. 98, 1889-1894. doi: 10.1073/pnas.98.4.1889

Truong, H. M., Lu, Z., Kutish, G. F., Galeota, J., Osorio, F. A., and Pattnaik, A. K. (2004). A highly pathogenic porcine reproductive and respiratory syndrome virus generated from an infectious cDNA clone retains the in vivo virulence and transmissibility properties of the parental virus. Virology 325, 308-319. doi: 10.1016/j.virol.2004.04.046

van der Hoeven, B., Oudshoorn, D., Koster, A. J., Snijder, E. J., Kikkert, M., and Barcena, M. (2016). Biogenesis and architecture of arterivirus replication organelles. Virus Res. 220, 70-90. doi: 10.1016/j.virusres.2016.04.001

van Hemert, M. J., de Wilde, A. H., Gorbalenya, A. E., and Snijder, E. J. (2008). The in vitro RNA synthesizing activity of the isolated arterivirus replication/transcription complex is dependent on a host factor. J. Biol. Chem. 283, 16525-16536. doi: 10.1074/jbc.M708136200

Xue, F., Sun, Y., Yan, L., Zhao, C., Chen, J., Bartlam, M., et al. (2010). The crystal structure of porcine reproductive and respiratory syndrome virus nonstructural protein Nsplbeta reveals a novel metal-dependent nuclease. J. Virol. 84, 6461-6471. doi: 10.1128/JVI.00301-10

Yang, L., Wang, R., Yang, S., Ma, Z., Lin, S., Nan, Y., et al. (2018). Karyopherin alpha6 is required for the replication of porcine reproductive and respiratory syndrome virus and Zika virus. J. Virol. 92, e72-e18. doi: 10.1128/JVI.00072-18

Yu, Y., Wang, R., Nan, Y., Zhang, L., and Zhang, Y. (2013). Induction of STAT1 phosphorylation at serine 727 and expression of proinflammatory cytokines by porcine reproductive and respiratory syndrome virus. PLoS One 8:e61967. doi: 10.1371/journal.pone.0061967

Zhang, M., Cao, Z., Xie, J., Zhu, W., Zhou, P., Gu, H., et al. (2013). Mutagenesis analysis of porcine reproductive and respiratory syndrome virus nonstructural protein 7. Virus Genes 47, 467-477. doi: 10.1007/s11262-013-0957-4

Zhao, S., Ge, X., Wang, X., Liu, A., Guo, X., Zhou, L., et al. (2015). The DEAD-box RNA helicase 5 positively regulates the replication of porcine reproductive and respiratory syndrome virus by interacting with viral Nsp9 in vitro. Virus Res. 195, 217-224. doi: 10.1016/j.virusres.2014.10.021

Conflict of Interest Statement: The authors declare that the research was conducted in the absence of any commercial or financial relationships that could be construed as a potential conflict of interest.

Copyright (c) 2018 Nan, Lan, Tian, Dong, Tian, Liu, Xu and Chen. This is an openaccess article distributed under the terms of the Creative Commons Attribution License (CC BY). The use, distribution or reproduction in other forums is permitted, provided the original author(s) and the copyright owner are credited and that the original publication in this journal is cited, in accordance with accepted academic practice. No use, distribution or reproduction is permitted which does not comply with these terms. 